\title{
Resultados de investigación para el sector de la salud en Cuba, 2006
}

\author{
Results of the health research work in Cuba, 2006
}

\author{
Niviola Cabrera Cruz'; I leana Quiñones de La Rosa"; Adolfo Álvarez \\ Blanco"'I; Freddy Gómez Martínez'v
}

'Especialista de II Grado en Epidemiología. Ministerio de Salud Pública. La Habana, Cuba.

"Especialista de I Grado en Farmacología. Ministerio de Salud Pública. La Habana, Cuba.

II'Especialista de II Grado en Organización y Administración de Salud. Ministerio de Salud Pública. La Habana, Cuba.

IVEspecialista de I Grado en Higiene y Epidemiología. Ministerio de Salud Pública. La Habana, Cuba.

\section{RESUMEN}

Con el presente trabajo se ha pretendido resumir en un grupo de indicadores y en seis tablas las principales características de la actividad científico-técnica desarrollada por las Entidades de Ciencia e Innovación Tecnológica y otras unidades del Sistema Nacional de Salud durante el año 2006. La mayoría de los resultados que aquí se incluyen se han obtenido a partir de proyectos de investigación ramales, territoriales e institucionales realizados por investigadores, profesores o profesionales en general y que han tributado a diversas salidas de las cuales se han incluido fundamentalmente publicaciones nacionales e internacionales, premios, tesis de maestría, doctorado o terminación de especialidad, patentes obtenidas o en proceso. Se hace un análisis de la información brindada por las unidades y se relaciona los resultados obtenidos con su alcance nacional, territorial y/o local en base a las proyecciones estratégicas del Ministerio de Salud Pública así como en función del impacto que representan para el Sistema Nacional de Salud, elementos de mucho interés en el momento de tomar decisiones para la generalización y el financiamiento, entre otras acciones. Se encontró que a pesar del incremento de la actividad científico-técnica en el sector de la salud en los últimos años, no existe 
una correspondencia adecuada con la utilización eficiente y eficaz del potencial científico que se dispone. Se pretende e interesa perfeccionar y dar continuidad a este tipo de trabajo a partir de la información que cada año brindan las instituciones científicas del país.

Palabras clave: Actividad científico-técnica, sector de la salud, Cuba.

\begin{abstract}
The present paper was aimed at presenting the main characteristics of the scientific and technical work performed by scientific and technological innovation entities as well as by other units within the national healthcare system in the year 2006. This work was summarized in a group of indicators and six tables. Most of the results mentioned here derived from branch, territorial and institutional research projects that have been conducted by researchers, professors and health professionals in general and that have led to several outputs of which national and international publications, prizes, master's course, PhD or specialty theses, approved patents and so have been mainly included in this paper. An analysis was made of the information provided by the entities and units, and the achieved results with their national, territorial and/or local scope in line with the strategic goals of the Ministry of Public Health and in terms of their impact on the national health care system were listed. The aforementioned elements are very important at the time of making decisions for generalization and financing of projects, among other actions. It was found that despite the rise of the technical and scientific activity in the health sector in the last few years, available scientific potentialities have not been yet efficiently and effectively utilized. It is intended to improve and to continue producing this type of paper on the basis of the information that the domestic scientific institutions report each year.
\end{abstract}

Key words: Health research work, Cuba.

\title{
EL SISTEMA
}

El Sistema de Ciencia e Innovación Tecnológica en el sector de la salud está conformado por un amplio conjunto de instituciones y recursos cuya finalidad principal es la producción, adaptación, transmisión, difusión y uso de los resultados científicos y técnicos.

En los últimos 10 años se han aplicado diversos mecanismos encaminados a garantizar el cumplimiento de las políticas de desarrollo y fortalecimiento de la actividad científica en el sector de acuerdo a las necesidades sociales y económicas, nacionales y locales. A partir de 1998 y como resultado de la Inspección Gubernamental al Ministerio de Salud Pública (MINSAP) se propuso por la Dirección del Ministerio de Ciencia, Tecnología y Medio Ambiente (CITMA), y de hecho se implementó en el organismo, el Sistema de Ciencia e Innovación Tecnológica. A partir de ese momento se observa un cambio de tendencia muy favorable para la 
actividad científico técnica y de innovación tecnológica, pero aún limitada si se tienen en cuenta las potencialidades del sector.

Es importante señalar que este salto se ha debido a un largo proceso. Desde entonces y hasta la fecha, se han realizado siete convocatorias para proyectos a programas ramales, han aumentado los temas y el número de proyectos presentados, ha crecido el presupuesto en moneda nacional y la respuesta por parte de los grupos de investigadores y profesionales en general ha sido mayor. Todo esto se expresa en un conjunto de indicadores que muestran la productividad científica en el MINSAP y explican el escenario actual. Entre ellos se destaca los siguientes:

1. Incremento del número de entidades de ciencia e innovación tecnológica, que según el Registro Nacional de Entidades de Ciencia e Innovación Tecnológica (EnClT) del $\mathrm{CITMA}^{1}$ suman ya 37 en el MINSAP, de ellas:

Según categorías:

Institutos y centros de investigación

Centros de servicios científico

tecnológicos

8

Unidades de desarrollo científico

tecnológico

8

Según provincias:

Ciudad de La Habana

28

Cienfuegos

Villa Clara

Camagüey

Holguín

1

Santiago de Cuba

Según nivel de atención:

Institutos

Hospitales

Policlínicos

Otros

2. El Sistema Nacional de Salud (SNS) tiene organizadas las actividades de investigación-desarrollo e innovación tecnológica mediante la presentación de proyectos, en programas nacionales, ramales y territoriales. En los primeros, aunque en un número limitado, se trabaja en proyectos vinculados a los programas de productos biotecnológicos y de la industria médico farmacéutica y de vacunas humanas y veterinarias. 
3. Se incrementa a 13 el número de programas científico-técnicos de carácter ramal al aprobarse por el CITMA los Programas Ramales de Cáncer, Atención Médica Integral a la Familia, Seguridad, Protección y Vigilancia para la Prevención de la Salud Humana, e Informática en Salud. Para la propuesta y activación de los programas de ciencia y técnica (C-T) en el MINSAP, se cumple lo que está establecido en la Resolución Ministerial (RM) № 85/2003 "Sistema de Programas y Proyectos". Estos constituyen un instrumento para la planificación, organización, financiamiento y control de las actividades de ciencia e innovación tecnológica, orientadas a resolver los problemas identificados en las prioridades. El sistema se conforma a partir de la definición de objetivos y resultados a alcanzar en un plazo determinado y se ejecuta mediante proyectos. $^{2}$

4. Se eleva el número de proyectos presentados a los programas científico-técnicos en cada convocatoria y en ejecución:

- La convocatoria a programas ramales de 2007 registró 560 proyectos a evaluar y en ejecución 1112 (mayo del 2007).

- El número de proyectos territoriales asciende a 281.

- El registro de proyectos institucionales como categoría transitoria es de 3096.

5. Se eleva el potencial científico, el que incluye a investigadores y docentes con alguna de las categorías correspondientes y otros profesionales que le dan soporte a la actividad científica.

Profesionales con categoría

científica

Doctores en Ciencias

Profesionales con categoría

docente

Aspirantes a Grados.
1564

604

22643

250

6. El promedio de edad entre los investigadores disminuye de 45 años en el 2000 a 39 en el 2007.

7. Se fortalece el programa de formación de doctores en todo el sector y se implementa una estrategia nacional para el seguimiento y control de esta actividad.

8. Se integra las estrategias de posgrado, servicios ambulatorios y ciencia e innovación tecnológica, lo que conduce a un plan de acción mucho más completo.

9. Aumenta el financiamiento para la actividad científica en la Rama 10 (Evaluación y Tecnologías Sanitarias), y, en particular, para proyectos:

1998 37,5 millones de pesos

2006 69,7 millones de pesos

2007 98,0 millones de pesos

10. Se establece una cultura de premios y reconocimientos, así como un cronograma para el seguimiento de los resultados con posibilidades de publicar, 
generalizar o para optar a premios en las diferentes convocatorias nacionales que ofrecen los Ministerios de Educación Superior (MES), Ciencia y Tecnología (CITMA), Academia de Ciencias de Cuba, MINSAP, Brigadas Técnicas Juveniles (BTJ), Asociación Nacional de Innovadores y Racionalizadores (ANIR) y Forum de Ciencia y Técnica.

En los dos últimos años la Dirección de Ciencia y Técnica del MI NSAP en consideración con los elementos de la planificación estratégica elaboró sus estrategias y objetivos priorizados, extendiendo el Sistema de Ciencia e Innovación Tecnológica a otras unidades del SNS, en particular a la atención primaria y a un grupo importantes de hospitales, con la incorporación de la investigación y la innovación a su accionar como actores sociales del Sistema. Este nuevo escenario significa un gran reto para todos los actores del proceso, y una estrategia efectiva en este sentido, deberá contemplar la coordinación de acciones entre todas las áreas del ministerio, de todos los colectivos científicos, de los expertos de los programas científicos y de los que deciden, ya que el registro, seguimiento y evaluación de los proyectos de investigación guardarían una legitimación mayor.

Hoy la investigación dentro del sector presenta otras formas de retorno social además del que se obtiene por la generación de un nuevo conocimiento que se pone al alcance de la comunidad científica. En parte, este retorno social viene dado por estar la investigación orientada a los problemas y necesidades tanto de la población como del sistema de salud. No cabe duda en este sentido, de que las convocatorias para la presentación de proyectos a los programas ramales de salud son más efectivas y responden más a ese objetivo que aquellos otros, donde el interés particular del investigador o de la institución, son los que priman.

Según el Artículo 4 de la RM № 85 del 2003 "Sistema de programas y Proyectos" los proyectos constituyen la célula básica para el financiamiento de las actividades de investigación-desarrollo, innovación, servicios científico-técnicos, actividades productivas vinculadas a la ciencia, así como actividades de interfase, gestión, y otras, que materializan los objetivos y resultados de los programas. ${ }^{2}$ Sus resultados se expresan en beneficios específicos y con un impacto en lo científico, económico, político, social, cultural, tecnológico, comercial o ambiental.

En el año 1996 la Dirección de Ciencia y Técnica del Ministerio de Salud Pública en correspondencia con la política científica del país y lo establecido por el Organismo Rector de la Ciencia, Tecnología y Medio Ambiente, puso en marcha una convocatoria para proyectos de investigación en las instituciones de salud del país que debían corresponderse con los programas ramales aprobados y vigentes en ese momento. Estos programas respondían en su mayor parte, a las necesidades del SNS. Considerando el punto de referencia inicial en aquel 1996, se puede decir que 10 años después, la diferencia es ostensible y ya no sólo se habla de proyectos sino de resultados de la ciencia y la técnica.

Según el Manual de Normas y Procedimientos del Sistema de Programas y Proyectos, para cada proyecto es preciso lograr la certificación de resultados, que no es más que la evaluación periódica del cumplimiento de los resultados que se obtienen por el proyecto en cuestión. ${ }^{3}$ Para los programas científico-técnico de carácter nacional, estos se proponen al secretario de programa por el director de la entidad ejecutora principal y se aprueba por el grupo de expertos del programa, pero en el caso del sector de la salud, se propone directamente a la Dirección de Ciencia y Técnica del organismo, la que con la asesoría de los respectivos expertos de los programas a los cuales pertenecen, los evalúan y certifican que cumplen con esa condición. Todo este proceso se efectúa en los meses de octubre-noviembre. ${ }^{3}$ 
La organización durante estos años de las actividades científicas y tecnológicas en el sector de la salud estructuradas en 13 programas ramales han permitido obtener resultados importantes, que han contribuido al bienestar de la población cubana, a mejorar los indicadores de salud y la calidad de los servicios. Algunos de estos programas se vienen desarrollando desde hace varios años, mientras que otros se han incorporado recientemente dadas las prioridades de la ciencia en esos campos (recuadro)

Según la Ley de Ciencia y Técnica en su Artículo 2, uno de los principios básicos en que se sustenta el ejercicio de las actividades científicas y tecnológicas en la República de Cuba, son los resultados obtenidos de ellas, los cuales deben ser aplicados en la práctica social de forma rápida y eficaz, teniendo en cuenta los criterios de utilidad, viabilidad y factibilidad. ${ }^{4}$

La convocatoria 2004-2006 se cierra con la presentación de una memoria científica en la que se dan a conocer los resultados de mayor relevancia en los servicios de salud y en el cuadro epidemiológico del país. En ella se recoge los resultados más importantes de las investigaciones realizadas, así como la producción científica que, en forma de publicaciones u otros tipos de salidas, se han derivado de esas convocatorias.

Estos resultados pueden medirse en términos muy concretos, tales como: evaluación de nuevas vacunas, medicamentos, biomateriales, procederes de diagnóstico, equipos médicos y servicios especializados de alto valor agregado.

\section{RESULTADOS}

La obtención de estos resultados ha sido posible entre otros factores, por el potencial humano que dispone el SNS, su principal capital, así como la participación de la comunidad y la interacción de todos los sectores del país. En este sentido es responsabilidad de los directivos utilizar los recursos humanos y materiales con mayor eficiencia y eficacia, y es en este contexto, que la Dirección de Ciencia y Técnica ha ganado conciencia de la necesidad de articular la actividad de ciencia e innovación tecnológica con los objetivos sociales y económicos principales, ya que los mismos, además de requerir la participación de todos los sectores, de los órganos de gobierno y de todo el pueblo, también requieren de la ejecución de estrategias bien definidas en ciencia, tecnología y protección del medio ambiente, como elementos decisivos para la elevación de la eficiencia del sector en la prestación de los servicios sanitarios y en el mejoramiento progresivo de los indicadores de salud del pueblo cubano.

A continuación se analiza los 357 resultados relevantes obtenidos en el año 2006 informados por todos los territorios y las entidades de ciencia e innovación tecnológica (EnCIT). Se utiliza este concepto para aquellos resultados alcanzados por los colectivos de autores que hubieran tributado a publicaciones nacionales e internacionales, premios, tesis de maestría, doctorado o terminación de especialidad, patentes obtenidas o en proceso; siempre vinculados a proyectos ramales o territoriales, fundamentalmente.

El interés por recopilar la información que permita obtener conocimientos sobre los perfiles y las tendencias de la investigación en salud, mediante los proyectos y resultados de la investigación, ha sido estudiado por diferentes autores los que han identificado algunos indicadores cualitativos de la producción científica, 
relacionándolos con la disciplina y el área temática, entre otros. ${ }^{5}$ En este informe se distribuyen los resultados teniendo en cuenta:

- El tipo de institución que lo propuso,

- el aporte de resultados de cada territorio y $\mathrm{EnClT}$,

- el alcance nacional, territorial y local de los resultados,

- su relación con las proyecciones estratégicas del MI NSAP,

- salidas específicas y otras salidas relacionadas fundamentalmente con la productividad científica e,

- impactos.

La mayor cantidad de resultados presentados en este informe (145), proviene de los centros de enseñanza médica superior (CEMS) que representan el 40,6\%. Tributan a este grupo otras unidades territoriales como son los hospitales y policlínicos. Les siguen los centros de investigación o EnCIT con 121 para el 33,9 \% y los institutos de investigación (91) con 25,5\%.

Si se analiza el aporte de resultados por cada territorio, excluyendo entidades de ciencia y técnica, se destaca las provincias de Ciudad de La Habana $(16,5 \%)$, Santiago de Cuba (15\%), Cienfuegos (10\%) y Holguín (9,4 \%). Como se puede apreciar, el aporte de resultados relevantes por territorios es bajo aún, provincias como Matanzas, La Habana, Las Tunas, Ciego de Ávila e I sla de la Juventud aportaron poco al total de resultados alcanzados (14\%) (tabla 1).

Las EnCIT de Ciudad Habana aportaron 176 resultados destacándose los Institutos del Área de Higiene y Epidemiología (31,2 \%) y Centros como el Centro de Investigación y Desarrollo de Medicamentos (CIDEM), elCentro de Química Farmacéutica (CQF) y el Centro de Control Estatal de Medicamentos (CECMED) $(23,0 \%)$ en conjunto.

Las EnCIT de los territorios, de más reciente incorporación al Sistema, aportaron 36 resultados, el mayor porcentaje de esos aportes correspondió al Centro de Investigación y Rehabilitación de las Ataxias Hereditarias de Holguín (33,3 \%), el Centro de Toxicología y Biomedicina de Santiago de Cuba $(22,0 \%$ ) y el Hospital "Gustavo Aldereguía Lima" de Cienfuegos (16,65\%) (tabla 2).

Considerando el alcance de los resultados se pudo constatar que de los 181 resultados aportados por los territorios más del $50 \%$ tuvieron un alcance territorial en correspondencia con el papel que deben jugar en la solución de los problemas de salud a nivel local. Tuvieron un alcance nacional el 34,8 \% de los resultados y el $13,2 \%$ tuvo un alcance local. El comportamiento de esta distribución en las EnCIT fue diferente. Casi el $90 \%$ de los resultados tuvo un alcance nacional, en correspondencia con su misión esencial (tablas 3 y $\underline{4}$ ).

En el análisis de los resultados en correspondencia con las Proyecciones Estratégicas del MINSAP, se observa que el principal destino de los resultados va dirigido a las enfermedades crónicas no transmisibles y otros daños a la salud los cuales aportaron el 32,2 \%, entre ellos 25 al programa de control de cáncer, 18 a las cardiopatías isquémicas, 12 a la enfermedad cerebrovascular, 11 a la enfermedad renal crónica y 10 a la hipertensión arterial. Tributaron al conocimiento de los accidentes y agresiones 14 resultados científicos (tabla 5). A las enfermedades transmisibles emergentes y reemergentes el $14 \%$, y a la atención a grupos especiales el $12 \%$.

A grandes rasgos se puede deducir que la investigación en salud ha abordado las enfermedades de mayor prevalencia en Cuba, sin embargo, existe una mayor 
concentración en los aspectos que han manifestado una larga trayectoria de investigación como son las enfermedades infecciosas y parasitarias y las de origen vascular.

Hay un grupo de resultados que no se incluyeron en ninguna de las áreas priorizadas al ser resultados básicos en los que aún no fue posible identificar a cuál problema de salud tributarán.

En relación con las salidas específicas, el mayor porcentaje de los resultados estuvo dirigido hacia los Programas de Prevención y Atención Sanitaria con el 36,2 \%, así como a Nuevas Tecnologías y Procederes Diagnósticos y Terapéuticos el 29,2 \%. Para la actividad docente fueron presentados para su edición un grupo de importantes libros y otros materiales docentes que representan el 15, 1 \% (tabla 6).

Si se analiza otras salidas relacionadas por ejemplo con la producción científica es de destacar que se presentaron para su publicación 848 trabajos, 345 optaron por premios territoriales y nacionales. Solamente el $23 \%$ tributó a tesis de maestría, $14,2 \%$ a doctorados y el $21,3 \%$ a tesis de terminación de especialidad.

En relación con los impactos el mayor porcentaje de los resultados tuvo un impacto científico con el $82 \%$, impacto social el $60 \%$, económico el $20 \%$ y tecnológico el $11,5 \%$.

Se puede concluir que a pesar del incremento de la actividad científico técnica en el sector en los últimos años, no existe una correspondencia adecuada con el potencial científico que se dispone. No todas las áreas priorizadas de la salud tienen cubiertas sus prioridades con la actividad científico técnica. El desarrollo científico técnico y la obtención de resultados no es uniforme en todas las EnCIT y provincias del país y aún es insuficiente el porcentaje de resultados que tributan al desarrollo de capacidades.

\section{REFERENCI AS BI BLI OGRÁFICAS}

1. Resolución 78/2003 del Ministerio de Ciencia, Tecnología y Medio Ambiente sobre el Registro Nacional de Entidades de Ciencia e Innovación Tecnológica. La Habana: Ministerio de Ciencia, Tecnología y Medio Ambiente; 2003.

2. Resolución 85/2003 del Ministerio de Ciencia, Tecnología y Medio Ambiente sobre el Reglamento del Sistema de Programas y Proyectos. La Habana: Ministerio de Ciencia, Tecnología y Medio Ambiente; 2003.

3. Ministerio de Ciencia Tecnología y Medio Ambiente. Manual de Normas y Procedimientos para la Gestión de Programas y Proyectos. La Habana: Ministerio de Ciencia Tecnología y Medio Ambiente; Marzo 2005.

4. Ley $N^{\circ} 75$. Ley de Ciencia y Técnica de la Republica de Cuba. 21 de diciembre de 1994.

5. Organización Panamericana de la Salud. Plan de desarrollo para proyectos de investigación sobre la situación de la investigación en salud en América Latina.

Washington, D.C.: OPS; Septiembre 1988. 
Recibido: 10 de agosto de 2007.

Aprobado: 5 de septiembre de 2007.

Niviola Cabrera Cruz. Dirección de Ciencia y Técnica. Ministerio de Salud Pública. La Habana, Cuba. E-mail: ncc@infomed.sld.cu

Recuadro. Programas ramales de salud

\author{
1. Salud reproductiva y Materno infantil \\ 2. Calidad de vida \\ 3. Enfermedades transmisibles \\ 4. Enfermedades no transmisibles y accidentes \\ 5. Cáncer \\ 6. Atención Médica Integral a la..Familią \\ 7. Investigación en Sistemas y Servicios de Salud \\ 8. Medicamentos y Medios Diagnósticos \\ 9. Medicina Natural y Tradicional \\ 10. Evaluación de Tecnologías Sanitarias \\ 11. Longevidad satisfactoria y Esperanza de vida (antes Adulto mayor) \\ 12. Informática en salud \\ 13. Seguridad, Protección y vigilancia para la.Prevención de la. Salud Humana
}


Tabla 1. Rresultados de ciencia y técnica del año 2006 según provincias. MINSAP*

\begin{tabular}{|l|c|c|}
\hline \multicolumn{1}{|c|}{ Provincias } & No. & \% \\
\hline Pinar de Río & 10 & 5,5 \\
\hline Habana & 6 & 3,3 \\
\hline Ciudad de la Habana & 30 & 16,5 \\
\hline Matanzas & 4 & 2,2 \\
\hline Villa Clara & 16 & 9 \\
\hline Cienfuegos & 18 & 10 \\
\hline Sancti Spíritus & 8 & 4,4 \\
\hline Ciego de Avila & 6 & 3,3 \\
\hline Camaguey & 9 & 5 \\
\hline Las Tunas & 4 & 2,2 \\
\hline Holguín & 17 & 9,3 \\
\hline Granma & 9 & 5 \\
\hline Santiago de Cuba & 12 & 15 \\
\hline Guantánamo & 5 & 6,6 \\
\hline Isla de la Juventud & 181 & 100,0 \\
\hline Total & & \\
\hline
\end{tabular}

*Excluye entidades de ciencia y técnica.

Tabla 2. Resultados del año 2006 por entidades de ciencia e innovación tecnológica de otras provincias. MINSAP

\begin{tabular}{|l|c|c|}
\hline EnCIT- provincias & No. & $\%$ \\
\hline Hosp. "Gustavo Aldereguía Lima" - Cienfuegos & 6 & 16,5 \\
\hline Unidad de Toxicología Experimental - Villa Clara & 4 & 11,1 \\
\hline Cardiocentro - Villa Clara & 4 & 11,1 \\
\hline Centro de Inmunología y Productos Biológicos - Camaguey & 1 & 3,0 \\
\hline $\begin{array}{l}\text { Centro de Desarrollo Ciencias Sociales y Humanísticas - } \\
\text { Camaguey }\end{array}$ & 1 & 3,0 \\
\hline $\begin{array}{l}\text { Centro Investigación Rehabilitación Ataxias Hereditarias - } \\
\text { Holguín }\end{array}$ & 12 & 33,3 \\
\hline Centro de Toxicología Experimental - Santiago Cuba & 8 & 22,0 \\
\hline Total & 36 & 100,0 \\
\hline
\end{tabular}

EnCIT: entidades de ciencia e innovación tecnológica. 
Tabla 3. Resultados del año 2006 según su alcance nacional, territorial o local. MINSAP

\begin{tabular}{|c|c|c|c|c|}
\hline & \multicolumn{4}{|c|}{ Alcance } \\
\hline & Nacional & Territorial & Local & Total \\
\hline Pinar de Río & - & 7 & 3 & 10 \\
\hline Habana Este & 1 & 5 & - & 6 \\
\hline Ciudad de la Habana & 19 & 9 & 2 & 30 \\
\hline Matanzas & - & 4 & - & 4 \\
\hline Villa Clara & 11 & 5 & - & 16 \\
\hline Sancti Spíritus & 5 & 12 & 1 & 18 \\
\hline Cienfuegos & - & 8 & - & 8 \\
\hline Ciego de Ávila & 2 & 3 & 1 & 6 \\
\hline Camagüey & 4 & 5 & - & 9 \\
\hline Las Tunas & 1 & 2 & 1 & 4 \\
\hline Holguín & 13 & 4 & - & 17 \\
\hline Granma & - & 4 & 5 & 9 \\
\hline Santiago de Cuba & 6 & 14 & 7 & 27 \\
\hline Guantánamo & 1 & 10 & 1 & 12 \\
\hline Isla de la Juventud & - & 2 & 3 & 5 \\
\hline Total & 63 & 94 & 24 & 181 \\
\hline
\end{tabular}


Tabla 4. Resultados del año 2006 según su alcance nacional, territorial o local -EnCIT-C. de La. Habana, MINSAP

\begin{tabular}{|c|c|c|c|c|}
\hline & \multicolumn{4}{|c|}{ Alcance } \\
\hline & Nacional & Territorial & Local & Total \\
\hline Inst.de Hematología & 1 & - & - & 1 \\
\hline $\begin{array}{l}\text { Inst. de } \\
\text { Endocrinología }\end{array}$ & 4 & 1 & - & 5 \\
\hline $\begin{array}{l}\text { Inst. de } \\
\text { Gastroenterología }\end{array}$ & 5 & - & - & 5 \\
\hline Inst. de Nefrología & 7 & - & - & 7 \\
\hline $\begin{array}{l}\text { Inst. de Cardiología y } \\
\text { Cirugía Cardiovascular }\end{array}$ & 2 & - & - & 2 \\
\hline $\begin{array}{l}\text { Inst. de Angiología y } \\
\text { Cirugía Vascular }\end{array}$ & 4 & 1 & - & 5 \\
\hline $\begin{array}{l}\text { Inst. de Neurología y } \\
\text { Neurocirugía }\end{array}$ & 3 & - & - & 3 \\
\hline $\begin{array}{l}\text { Inst. de Oncología y } \\
\text { Radiobiología }\end{array}$ & 8 & - & - & 8 \\
\hline $\begin{array}{l}\text { Inst.Higiene,Epidemiología } \\
\text { y Microbiología }\end{array}$ & 15 & 1 & 1 & 17 \\
\hline $\begin{array}{l}\text { Inst.de Nutrición e } \\
\text { Higiene de los Almentos }\end{array}$ & 9 & 2 & 3 & 14 \\
\hline $\begin{array}{l}\text { Inst. de Salud de los } \\
\text { Trabajadores }\end{array}$ & 7 & 2 & - & 9 \\
\hline Inst. Pedro Kouri & 13 & 2 & - & 15 \\
\hline Total & 78 & 9 & 4 & 91 \\
\hline
\end{tabular}

EnCIT: entidades de ciencia e innovación tecnológica.

Tabla 5. Resultados del año 2006 relacionados con las áreas priorizadas de las proyecciones estratégicas de la salud. MINSAP

\begin{tabular}{|l|c|c|}
\hline \multicolumn{1}{|c|}{ Proyecciones estratégicas } & No. & \% \\
\hline Factores relacionados con el ambiente & 14 & 4 \\
\hline Factores relacionados con el comportamiento & 18 & 5 \\
\hline $\begin{array}{l}\text { Enfermedades no transmisibles y otros daños a la } \\
\text { salud }\end{array}$ & 115 & 32,2 \\
\hline Enfermedades buco dentales & 16 & 4,5 \\
\hline $\begin{array}{l}\text { Enfermedades transmisibles, emergentes, } \\
\text { reemergentes }\end{array}$ & 50 & 14 \\
\hline Discapacidad & 4 & 1,1 \\
\hline Ambientes especiales & 12 & 3,4 \\
\hline Grupos especiales & 43 & 12 \\
\hline Otros & 85 & 24 \\
\hline Total & 357 & 100,0 \\
\hline
\end{tabular}


Tabla 6. Resultados del año 2006 según salidas específicas. MINSAP

\begin{tabular}{|c|c|c|}
\hline Salidas especificas & No. & $\%$ \\
\hline $\begin{array}{l}\text { Nuevos registros y evaluaciones de } \\
\text { medicamentos }\end{array}$ & 47 & 13,1 \\
\hline $\begin{array}{l}\text { Nuevas tecnologías y procederes } \\
\text { diagnósticos, terapéuticos. }\end{array}$ & 104 & 29,2 \\
\hline Programas de prevención y atención & 129 & 36,2 \\
\hline Materiales para la docencia & 54 & 15,1 \\
\hline Manuales o guías & 23 & 6,4 \\
\hline Total & 357 & 100,0 \\
\hline
\end{tabular}

